\title{
THE EFFECT OF HACCP SYSTEM ON VARIOUS MICROBIOLOGICAL HAZARDS IN CHEESE FACTORIES
}

\author{
HANAA, F. SALAMA ${ }^{1}$; AMAL A. AL-SAID ${ }^{2}$ AND EL-ASUOTY, M.S ${ }^{1}$ \\ ${ }^{1}$ Senior Researcher, Food Hygiene Unit. \\ ${ }^{2}$ Senior Researcher, Mycology Unit. \\ Damanhour Provincial Lab., Animal Health Research Institute (AHRI), Agriculture Research Center \\ (ARC), Egypt.
}

Received: 5 October 2021; Accepted: 31 October 2021

\begin{abstract}
Due to the large number of complaints from some cheese producers in the El-Beheira Governorate, Egypt about the existence of problems in their cheese products, it was important to identify the causes of these problems and to provide recommendations and practical solutions. The Hazard Analysis and Critical Control Points (HACCP) system is a plantspecific and product-specific quality system that is one of the most significant systems in quality assurance and food safety programs. As a result, the application of the HACCP system plan in a small-scale cheese plant producing fresh damietta and ras cheese was investigated in order to determine the effect of the HACCP system application on microbiological hazards by determining the bacterial load of Critical Control Points (CCPs). The obtained results revealed that raw milk and equipment swabs were the most dangerous sources of contamination with different microorganisms. In addition, that the diversity of microorganisms and their counts in some raw materials, food handlers and food contact surfaces, besides, the processing conditions have an impact on the quality and safety of the product. The most prevalent fungal isolates from production line of both cheese factories were Rhodotorula followed by Candida spp, regarding to mould species penicillium and Asp. niger were the most prevalent mould species. Therefore, all instructions for implementing the HACCP system were prepared, including a flow diagram of processing processes, identifying risks, regulating at different CCPs by monitoring and corrective actions, confirming the HACCP plan, and keeping records.
\end{abstract}

Key words: HACCP, cheese, Coliforms, enterobacteriaceae, Staph. aureus, yeast \& mould.

\section{INTRODUCTION}

In Egypt, Damietta cheese is the most popular soft white pickled cheese variety

Corresponding author: Hanaa, F. Salama

E-mail address: hsalama689@gmail.com

Present address: Senior Researcher, Food Hygiene, Unit, Damanhour Provincial Lab., Animal Health Research Institute (AHRI), Agriculture Research Center (ARC), Egypt. and is named after the city and governorate of Damietta and it makes up about $75 \%$ of the cheese produced and consumed in Egypt (Zhang et al., 2003). Damietta cheese has a unique processing step in that it is salted at the beginning of the process; the salt is added directly to the raw milk; after salting and filtration, liquid animal rennet is added to have the coagulum in 2.5-3 hours; the curd is hooped into wooden moulds coated with mouslin; the filtrated salty 
whey is collected for pickling the damietta cheese. Tins of damietta cheese were left at room temperature for six months to achieve a well-ripened product with a sharp unique scent and acid flavour. The cheese had a somewhat firm texture and was given the name "Estambulli cheese" (Hamad, 2015).

Ras cheese is the national hard cheese type in Egypt. It is known in Egyptian markets as "Romi cheese". It is similar to the Greek variety "Kefalotyri cheese". It is made from raw cow milk or from a mixture of cow and buffalo milks (Abou-Donia, 2002). Different groups of microorganisms would be present in this cheese from the used raw milk and from the surrounding environment (Girgis et al., 1992). Some of these bacteria are beneficial to the development of flavour and body texture, while others are harmful and create flaws in the cheese. Molds and yeasts can contaminate it as a result of unregulated sanitary conditions. As a result, the action of this flora has an impact on the ultimate taste and texture of Ras cheese. (Ayad et al., 2004).

Dirty food contact surfaces and poor personal hygiene standards among food handlers are two of the leading sources of microbial contamination and proliferation in food items, according to several researchers (EFSA, 2007; WHO, 2007; Jones et al., 2008). Equipment, food contact surfaces, and food handlers' hands have been the primary causes of crosscontamination (Tsalo et al., 2007; FusterValls et al., 2008). Food contact surfaces, according to (Evans et al., 2004), can be a source of contamination if they are not properly cleaned or remain wet between cleaning and usage. Food handlers, according to (Walker et al., 2003), play a significant role in probable crosscontamination because they can be asymptomatic carriers of foodborne disease-causing bacteria.

The spoilage caused by fungal contamination of raw milk and cheese is manifested by the presence of many metabolic by-products, causing off-flavors, odors and visible changes in color and texture. Many yeasts growing in cheese resulting in yeasty tastes by producing alcohol and $\mathrm{CO} 2$, and some yeasts producing sulfides which causes egg odour. Penicillium spp. and Cladosporium spp. are commonly moulds growing in vacuumpackaged cheeses (Ledenbach and Marshall, 2009).

Microbiological hazards are the most common possible hazards in most dairy products, microbiological testing may often be required to verify that raw materials are delivered in agreement with local specifications and as a mean of monitoring for selecting and approving the suppliers. Total aerobic count, coliforms count, and total yeast and mould counts are used as indicators for the quality of cheese (McMeekin, 2003).

HACCP is a systematic preventive approach to food safety that focuses on physical, chemical, and biological dangers rather than completed product inspection. HACCP used in the food industry to identify potential food safety hazards and is applied at every stage of the food manufacturing and preparation process, including packing, distribution. HACCP encompassing all aspects of food industry including meat, poultry, seafood, and dairy, it starts from the farm to the fork (Grujic et al., 2010).

Swabs from cleaned surfaces, food workers' hands, and food contact surfaces can be used to sample the environment. Early detection of pathogens allows for appropriate action to be taken to remove the pathogen, increasing the operator's degree of safety. Environmental sampling is a method of ensuring that items comply with food safety regulations (Opiyo et al., 2013).

The aim of this research was monitoring efficiency of HACCP system set up in processing line of two different cheese factories (Damietta and Ras) through 
microbial analysis of final cheese products, raw milk, water supply, rennet enzyme, salt, air, curd, whey, and environmental samples (swabs) from workers' hands and cheese contact surfaces.

\section{MATERIALS AND METHODS}

\section{Collection of samples:}

A total 202 representative samples were collected along the production line of two separate cheese (damietta and ras) at ElBahaira Governorate. The samples were transported to the laboratory (Animal Health Research Institute, Damanhour Branch) as quickly as possible in an insulated icebox at $4 \pm 1{ }^{\circ} \mathrm{C}$. The samples were collected according to following table.

\begin{tabular}{lcl}
\hline Samples & Damietta cheese & Ras cheese \\
\hline Damietta cheese & 10 & 10 \\
\hline Raw milk & 10 & 10 \\
\hline Water supply & 10 & 10 \\
\hline Rennet enzyme & 10 & 10 \\
\hline Salt & 10 & 10 \\
\hline Air & 10 & 10 \\
\hline Curd & 10 & 10 \\
\hline Whey & 10 & 10 \\
\hline Swabs & 3 & \\
\hline Milk tank & 3 \\
\hline Equipment & 3 & 3 \\
\hline Food handlers & 3 & 3 \\
\hline Tables & 3 & 3 \\
\hline Walls & 3 & 3 \\
\hline Packaging Material & 3 & 3 \\
\hline Refrigerator & 3 & 3 \\
\hline Total & 101 & 101 \\
\hline The collected samples from each factory were divided into two parts, $1^{\text {st }}$ part for physical and \\
chemical examination, $2^{\text {nd }}$ part for microbiological examination.
\end{tabular}

2. Chemical and sanitary evaluation of collected damietta and ras cheese: Fat contents were tested using Rose-Gottlieb Method, (905.02); moisture content of milk and cheese and $\mathrm{pH}$ values were measured using glass electrode $\mathrm{pH}$ meter (Model 810) Fisher Scientific according to (AOAC, 2012). (APHA, 2004) was used to determine salt contents. Titratable acidity was measured at $\%$ of lactic acid according to (AOAC, 2000).

\section{Microbiological evaluation of damietta and ras cheese:}

\subsection{Preparation of serial dilutions:}

3.1.1. Curd and cheese samples according to (ISO, 6887/2010): Ten $\mathrm{g}$ of the sample under test were weighed and added to $90 \mathrm{ml}$ of Dipotassium hydrogen phosphate solution preheated to $45^{\circ} \mathrm{C}$ then blended at rotatory blender until cheese thoroughly dispersed (1 $\mathrm{min}$ to $2-5 \mathrm{~min}$ ) to provide the initial suspension.

\subsubsection{Liquid samples according to} (APHA, 1992): Ten $\mathrm{ml}$ of liquid samples under test were added to $90 \mathrm{ml}$ of sterile distilled water and thoroughly mixed to prepare initial suspension. 
3.1.3. Swab samples according to (ISO 18593:2004) using horizontal method for collecting swabs from environmental sampling (food contact surfaces and food handlers' hands or gloves), a sample area of $50 \mathrm{~cm}^{2}$ was used for surfaces and swabs covered $25 \mathrm{~cm} 2$ ( 5 by $5 \mathrm{~cm}$ square) of each food handler's hand.

3.2. Microbiological examination of cheese samples: The obtained results were calculated as $\mathrm{Cfu} / \mathrm{ml}, \mathrm{g}, \mathrm{cm}^{2}$ or $\mathrm{m}^{3}$ as recommended by (Awad and Mawla, 2012).

3.2.1. Total bacterial count according to (APHA, 2004) using plate count agar, incubated at $32 \pm 1{ }^{\circ} \mathrm{C}$ for $48 \pm 3 \mathrm{~h}$, the number was expressed as $\mathrm{cfu} / \mathrm{ml}, \mathrm{g}$, and $\mathrm{cm}^{2}$.

\subsubsection{Total Staphylococcus aureus count} according to (APHA, 2004), using BairdParker agar (BPA) (Oxoid), incubated at 35 $\pm 1^{\circ} \mathrm{C}$ for $45-48 \mathrm{~h}$. Coagulase production, catalase test, anaerobic utilization of glucose and mannitol, and Gram stain were used for more assurance according to (Henning et al., 2004).

\subsubsection{Enterobacteriaceae count} according to (ISO 21528-2: 2004) using violet, red bile glucose agar (VRBG) (Oxoid), incubated at $37^{\circ} \mathrm{C}$ for $24 \mathrm{~h} \pm 2 \mathrm{~h}$.

\subsubsection{Coliforms count according to} APHA, (2004) was done using violet red bile agar (VRB) (Oxoid), incubated at $37^{\circ} \mathrm{C}$ for $24 \mathrm{~h} \pm 2 \mathrm{~h}$, dark red colonies were recorded as coliforms.

3.2.5. Total yeast and mould count according to (ISO, 6611:2004) was carried out on Sabourad's dextrose agar medium supplemented with chloramphenicol and chlortetracycline ((100 mg of each) with final $\mathrm{pH} 6.6 \pm 0.2$, plates incubated at $25 \pm$ $2^{\circ} \mathrm{C}$ for 3-5 days as described by (Koburger, 1970), Mould as well as yeast were counted separately and recorded as total mould and yeast count/ml.

The isolated fungi were identified individually by macro- and microscopic characteristics according to (Samson, 1979), (Pitt and Hoching, 2009), by the lactophenol-cotton-blue stain which identifies mould by culture characteristics, morphology of hyphae cells, spores and fruiting bodies.

Yeast isolates were identified according to (Kriger, 1984) and (Tibor and Larry, 1996). by Gram stain, the yeast was identified on the basis colony morphological character (blastocondia and chlamydospores according to (Raper and Fennel, 1965) and (Frey et al., 1979). Germ tube test was used for differentiation between Candida species in which a very light suspension of yeast like organisms in $0.5-1.0 \mathrm{ml}$ of sterile rabbit serum can be used. Incubation was occurred at $37{ }^{\circ} \mathrm{C}$ for no longer than $3 \mathrm{hrs}$. then one drop of yeast- serum mixture was placed on a slide slip and was examined microscopically for germ tube production.

4. Statistical analysis: Data was expressed as mean \pm SEM using Statistical data analysis was carried out using SPSS 17.0 for windows (SPSS Inc, Chicago, IL, USA).

\section{RESULTS}

Table 1: Statistical analytical results of chemical examination for the examined damietta cheese samples.

\begin{tabular}{lccc}
\hline \multicolumn{1}{c}{ Parameter } & Minimum & Maximum & Mean \pm SEM \\
\hline Acidity $(\%)$ & 0.25 & 2.5 & $1.36 \pm 0.25$ \\
pH & 3.35 & 6.01 & $4.43 \pm 0.099$ \\
Salt $(\%)$ & 3.90 & 6.50 & $5.01 \pm 0.25$ \\
Moisture $(\%)$ & 33.05 & 57.15 & $43.27 \pm 0.37$ \\
Fat $(\%)$ & 16.02 & 22.61 & $18.96 \pm 0.25$ \\
\hline
\end{tabular}


Table 2: Statistical analytical results of microbiological evaluation for examined damietta cheese during processing steps of production line.

\begin{tabular}{cccccccccc}
\hline \multirow{2}{*}{ Control points } & \multicolumn{3}{c}{ TBC } & \multicolumn{2}{c}{ Staph. arueus } & \multicolumn{2}{c}{ Enterobacteriaceae } \\
\cline { 2 - 10 } & $\mathbf{N O}$ & $\mathbf{\%}$ & Mean \pm SEM & NO & $\%$ & Mean \pm SEM & NO & \% & Mean \pm SEM \\
\hline Damietta cheese & 7 & 70 & $5.57 \times 10^{4} \pm 1.07 \times 10^{4}$ & 7 & 70 & $1.06 \times 10^{4} \pm 0.82 \times 10^{4}$ & 0 & 0.0 & 0.0 \\
\hline Raw milk & 10 & 100 & $5.38 \times 10^{7} \pm 1.09 \times 10^{7}$ & 9 & 90 & $3.8 \times 10^{4} \pm 1.01 \times 10^{4}$ & 3 & 30 & $5.8 \times 10^{4} \pm 4.06 \times 10^{4}$ \\
\hline Water supply & 5 & 50 & $4.8 \times 10 \pm 1.1 \times 10$ & 0 & 0.0 & 0.0 & 0 & 0.0 & 0.0 \\
\hline Rennet enzyme & 5 & 50 & $4.4 \times 10^{2} \pm 1.3 \times 10^{2}$ & 0 & 0.0 & 0.0 & 0 & 0.0 & 0.0 \\
\hline Salt & 4 & 40 & $2.2 \times 10^{3} \pm 0.47 \times 10^{3}$ & 0 & 0.0 & 0.0 & 0 & 0.0 & 0.0 \\
\hline Air & 5 & 50 & $2.4 \times 10 \pm 1.5 \times 10$ & 0 & 0.0 & 0.0 & 0 & 0.0 & 0.0 \\
\hline Curd & 5 & 50 & $1.5 \times 10^{4} \pm 0.71 \times 10^{4}$ & 5 & 50 & $6.8 \times 10^{3} \pm 2.2 \times 10^{3}$ & 0 & 0.0 & 0.0 \\
\hline Whey & 5 & 50 & $3.6 \times 10 \pm 0.8 \times 10$ & 0 & 0.0 & 0.0 & 0 & 0.0 & 0.0 \\
\hline Swabs (Cfu/cm2) & & & & & & 0 & 0.0 & 0.0 \\
\hline Milk tank & 3 & 100 & $7.6 \times 10^{2} \pm 2.6 \times 10^{2}$ & 0 & 0.0 & 0.0 & 0 & $2.0 \times 10^{4} \pm 0.8 \times 10^{4}$ \\
\hline Equipment & 3 & 100 & $4.7 \times 10^{7} \pm 2.4 \times 10^{7}$ & 3 & 100 & $3.7 \times 10^{4} \pm 2.4 \times 10^{4}$ & 3 & 100 & $9.0 \times 10^{2} \pm 0.00$ \\
\hline Food handlers & 3 & 100 & $1.7 \times 10^{4} \pm 0.5 \times 10^{4}$ & 3 & 100 & $3.2 \times 10^{3} \pm 0.79 \times 10^{3}$ & 1 & 33.3 & 0.0 \\
\hline Tables & 3 & 100 & $5.6 \times 10^{4} \pm 1.85 \times 10^{4}$ & 0 & 0.0 & 0.0 & 0 & 0.0 & 0.0 \\
\hline Walls & 3 & 100 & $2.8 \times 10^{4} \pm 1.4 \times 10^{4}$ & 3 & 100 & $5.2 \times 10^{3} \pm 3.4 \times 10^{3}$ & 1 & 33.3 & $1.0 \times 10 \pm 0.0$ \\
\hline $\begin{array}{c}\text { Packaging } \\
\text { Material }\end{array}$ & 3 & 100 & $7.6 \times 10 \pm 2.4 \times 10$ & 0 & 0.0 & 0.0 & 0 & 0.0 & 0.0 \\
\hline Efrigerator & 3 & 100 & $6.0 \times 10^{2} \pm 3.6 \times 10^{2}$ & 0 & 0.0 & 0.0 & 0 & 0.0 & 0.0 \\
\hline
\end{tabular}

Table 3: Statistical analytical results of microbiological evaluation for examined damietta cheese during processing steps of cheese production line.

\begin{tabular}{|c|c|c|c|c|c|c|c|c|c|}
\hline \multirow{2}{*}{ Control points } & \multicolumn{3}{|c|}{ Total coliforms } & \multicolumn{3}{|c|}{ T. Yeast } & \multicolumn{3}{|c|}{ T. mould } \\
\hline & NO & $\%$ & Mean \pm SEM & NO & $\%$ & Mean \pm SEM & NO & $\%$ & Mean \pm SEM \\
\hline Damietta cheese & 0 & 0.0 & 0.0 & 5 & 50 & $\begin{array}{l}.14 \times 10^{4} \pm \\
1.27 \times 10^{4} \\
\end{array}$ & 2 & 20 & $\begin{array}{r}1.3 \times 10^{3} \pm \\
0.5 \times 10^{3} \\
\end{array}$ \\
\hline Raw milk & 3 & 30 & $\begin{array}{l}1.4 \times 10^{4} \pm \\
0.41 \times 10^{4} \\
\end{array}$ & 7 & 70 & $\begin{array}{c}2.04 \times 10^{4} \pm \\
0.56 \times 10^{4}\end{array}$ & 6 & 60 & $\begin{array}{l}6.8 \times 10^{3} \pm \\
1.68 \times 10^{3} \\
\end{array}$ \\
\hline Water supply & 0 & 0.0 & 0.0 & 1 & 10 & $\begin{array}{c}1.0 \times 10^{3} \pm \\
0.0\end{array}$ & 2 & 20 & $\begin{array}{l}1.5 \times 10^{3} \pm \\
0.5 \times 10^{3} \\
\end{array}$ \\
\hline Rennet enzyme & 0 & 0.0 & 0.0 & 0 & 0.0 & 0.0 & 4 & 40 & $\begin{array}{l}1.2 \times 10^{3} \pm \\
0.25 \times 10^{3} \\
\end{array}$ \\
\hline Salt & 0 & 0.0 & 0.0 & 0 & 0.0 & 0.0 & 0 & 0.0 & 0.0 \\
\hline Air & 0 & 0.0 & 0.0 & 0 & 0.0 & 0.0 & 3 & 30 & $\begin{array}{c}3.5 \times 10^{2} \pm \\
3.3 \times 10^{2} \\
\end{array}$ \\
\hline Curd & 0 & 0.0 & 0.0 & 3 & 30 & $\begin{array}{r}4.3 \times 10^{3} \pm \\
0.66 \times 10^{3} \\
\end{array}$ & 3 & 30 & $\begin{array}{l}1.3 \times 10^{3} \pm \\
0.33 \times 10^{3}\end{array}$ \\
\hline Whey & 0 & 0.0 & 0.0 & 0 & 0.0 & 0.0 & 2 & 20 & $\begin{array}{c}1.0 \times 10^{3} \pm \\
0.0\end{array}$ \\
\hline \multicolumn{10}{|l|}{ Swabs (Cfu/cm2) } \\
\hline Milk tank & 1 & 33.3 & $\begin{array}{c}1.2 \times 10^{3} \pm \\
0.0\end{array}$ & 2 & 66.6 & $\begin{array}{c}5.4 \times 10^{2} \pm \\
4.5 \times 10^{2} \\
\end{array}$ & 1 & 33.3 & $\begin{array}{c}2.0 \times 10 \pm \\
0.00 \\
\end{array}$ \\
\hline Equipment & 3 & 100 & $\begin{array}{r}2.7 \times 10^{5} \pm \\
1.8 \times 10^{5}\end{array}$ & 1 & 33.3 & $\begin{array}{c}4.0 \times 10^{4} \pm \\
0.00\end{array}$ & 2 & 66.6 & $\begin{array}{r}2.5 \times 10^{3} \pm \\
2.4 \times 10^{3}\end{array}$ \\
\hline Food handlers & 1 & 33.3 & $\begin{array}{c}2.2 \times 10^{3} \pm \\
0.0 \\
\end{array}$ & 0 & 0.0 & 0.0 & 1 & 33.3 & $\begin{array}{c}7.0 \times 10^{3} \pm \\
0.00 \\
\end{array}$ \\
\hline Tables & 0 & 0.0 & 0.0 & 0 & 0.0 & 0.0 & 0 & 0.0 & 0.0 \\
\hline Walls & 1 & 33.3 & $2.0 \times 10^{2} \pm 0.0$ & 1 & 33.3 & $\begin{array}{c}3.0 \times 10^{2} \pm \\
0.00\end{array}$ & 2 & 66.6 & $\begin{array}{r}4.5 \times 10^{3} \pm \\
1.5 \times 10^{3}\end{array}$ \\
\hline $\begin{array}{c}\text { Packaging } \\
\text { Material }\end{array}$ & 0 & 0.0 & 0.0 & 0 & 0.0 & 0.00 .0 & 0 & 0.0 & 0.0 \\
\hline Refrigerator & 0 & 0.0 & 0.0 & 0 & 0.0 & 0 & 0.0 & 0.0 & 0.0 \\
\hline
\end{tabular}


Table 4: Incidence of yeast and fungal isolates in examined damietta cheese during processing steps of cheese production line collected from different sources $(\mathrm{N}=101)$

\begin{tabular}{|c|c|c|c|c|c|c|c|c|c|c|c|c|}
\hline \multirow{3}{*}{ Control points } & \multicolumn{6}{|c|}{ Yeast species } & \multicolumn{6}{|c|}{ Mould species } \\
\hline & \multicolumn{2}{|c|}{ C. albicans } & \multicolumn{2}{|c|}{ C. tropical } & \multicolumn{2}{|c|}{ Rhodotorula } & \multicolumn{2}{|c|}{ A. flavus } & \multicolumn{2}{|c|}{ A. Niger } & \multicolumn{2}{|c|}{ Penicillium } \\
\hline & No & $\%$ & No & $\%$ & No & $\%$ & No & $\%$ & No & $\%$ & No & $\%$ \\
\hline Damietta cheese & 1 & 10 & 3 & 10 & 4 & 40 & 2 & 20 & 2 & 20 & 2 & 20 \\
\hline Raw milk & 2 & 20 & 3 & 30 & 5 & 50 & 3 & 30 & 4 & 40 & 2 & 20 \\
\hline Water supply & 0 & 0 & 1 & 10 & 1 & 10 & 1 & 10 & 2 & 20 & 1 & 10 \\
\hline Rennet enzyme & 0 & 0 & 0 & 0 & 0 & 0 & 0 & 0 & 2 & 20 & 3 & 30 \\
\hline Salt & 0 & 0 & 0 & 0 & 0 & 0 & 0 & 0 & 0 & 0 & 0 & 0 \\
\hline Air & 0 & 0 & 0 & 0 & 0 & 0 & 0 & 0 & 3 & 30 & 2 & 20 \\
\hline Curd & 0 & 0 & 2 & 20 & 3 & 30 & 0 & 0 & 3 & 30 & 3 & 30 \\
\hline Whey & 0 & 0 & 0 & 0 & 0 & 0 & 1 & 10 & 2 & 20 & 2 & 20 \\
\hline \multicolumn{13}{|l|}{ Swaps } \\
\hline Milk tank & 0 & 0 & 1 & 33.3 & 2 & 66.6 & 0 & 0 & 1 & 33.3 & 1 & 33.3 \\
\hline Equipment & 1 & 10 & 1 & 33.3 & 1 & 33.3 & 1 & 33.3 & 2 & 66.6 & 2 & 66.6 \\
\hline Food handlers & 0 & 0 & 0 & 0 & 0 & 0 & 0 & 0 & 1 & 33.3 & 1 & 33.3 \\
\hline Tables & 0 & 0 & 0 & 0 & 0 & 0 & 0 & 0 & 0 & 0 & 0 & 0 \\
\hline Walls & 0 & 0 & 1 & 33.3 & 1 & 33.3 & 0 & 0 & 2 & 66.6 & 2 & 66.6 \\
\hline Packaging Material & 0 & 0 & 0 & 0 & 0 & 0 & 0 & 0 & 0 & 0 & 0 & 0 \\
\hline Refrigerator & 0 & 0 & 0 & 0 & 0 & 0 & 0 & 0 & 0 & 0 & 0 & 0 \\
\hline
\end{tabular}

Table 5: Statistical analytical results of chemical examination for examined ras cheese samples.

\begin{tabular}{lccc}
\hline Parameter & Minimum & Maximum & Mean \pm SEM \\
\hline Acidity (\%) & 1.10 & 2.40 & $1.76 \pm 0.06$ \\
pH & 4.01 & 5.95 & $4.96 \pm 0.066$ \\
Salt $(\%)$ & 1.80 & 5.95 & $2.35 \pm 0.036$ \\
Moisture (\%) & 25.35 & 39.75 & $32.23 \pm 0.45$ \\
Fat $(\%)$ & 45.22 & 50.24 & $47.79 \pm 0.17$ \\
\hline
\end{tabular}

Table 6: Statistical analytical results of microbiological evaluation for examined ras cheese during processing steps of cheese production line.

\begin{tabular}{cccccccccc}
\hline \multirow{2}{*}{ Control points } & \multicolumn{3}{c}{ TBC } & \multicolumn{3}{c}{ Staph. aureus } & \multicolumn{2}{c}{ Enterobacteriaceae } \\
\cline { 2 - 10 } & NO & \% & Mean \pm SEM & NO & \% & Mean \pm SEM & NO & \% & Mean \pm SEM \\
\hline Ras cheese & 10 & 100 & $2.7 \times 10^{5} \pm 0.76 \times 10^{5}$ & 10 & 100 & $4.6 \times 10^{4} \pm 0.86 \times 10^{4}$ & 0 & 0.0 & 0.0 \\
\hline Raw milk & 10 & 100 & $1.3 \times 10^{7} \pm 0.39 \times 10^{7}$ & 9 & 90 & $1.8 \times 10^{4} \pm 0.71 \times 10^{4}$ & 3 & 30 & $3.4 \times 10^{5} \pm 0.30 \times 10^{5}$ \\
\hline Water supply & 7 & 70 & $3.8 \times 10 \pm 0.5 \times 10$ & 0 & 0.0 & 0.0 & 0 & 0.0 & 0.0 \\
\hline Rennet enzyme & 5 & 50 & $8.4 \times 10^{2} \pm 5.4 \times 10^{2}$ & 0 & 0.0 & 0.0 & 0 & 0.0 & 0.0 \\
\hline Salt & 3 & 30 & $1.0 \times 10^{3} \pm 0.57 \times 10^{3}$ & 0 & 0.0 & 0.0 & 0 & 0.0 & 0.0 \\
\hline Air & 4 & 40 & $1.7 \times 10 \pm 0.4 \times 10$ & 0 & 0.0 & 0.0 & 0 & 0.0 & 0.0 \\
\hline Curd & 7 & 70 & $5.0 \times 10^{2} \pm 1.1 \times 10^{2}$ & 0 & 0 & 0.0 & 0 & 0.0 & 0.0 \\
\hline Whey & 6 & 60 & $2.1 \times 10 \pm 0.4 \times 10$ & 0 & 0.0 & 0.0 & 0 & 0.0 & 0.0 \\
\hline Swabs (Cfu/cm2) & & & & & & & 0 & 0.0 & 0.0 \\
\hline Milk tank & 3 & 100 & $3.3 \times 10^{2} \pm 0.88 \times 10^{2}$ & 0 & 0.0 & 0.0 & 0 & 0.0 \\
\hline Equipment & 3 & 100 & $4.07 \times 10^{7} \pm 2.3 \times 10^{7}$ & 3 & 100 & $5.3 \times 10^{5} \pm 1.1 \times 10^{5}$ & 0 & 0.0 & $5.9 \times 10^{5} \pm 2.4 \times 10^{5}$ \\
\hline Food handlers & 3 & 100 & $1.2 \times 10^{4} \pm 0.14 \times 10^{4}$ & 3 & 100 & $1.4 \times 10^{3} \pm 0.29 \times 10^{3}$ & 3 & 100 & 0.0 \\
\hline Tables & 3 & 100 & $2.3 \times 10^{4} \pm 0.88 \times 10^{4}$ & 0 & 0.0 & 0.0 & 0 & 0.0 & 0.0 \\
\hline Walls & 3 & 100 & $1.1 \times 10^{4} \pm 0.23 \times 10^{4}$ & 3 & 100 & $1.3 \times 10^{4} \pm 0.96 \times 10^{4}$ & 0 & 0.0 & 0.0 \\
\hline $\begin{array}{c}\text { Packaging } \\
\text { Material }\end{array}$ & 3 & 100 & $2.0 \times 10 \pm 0.5 \times 10$ & 0 & 0.0 & 0.0 & 0 & 0.0 & 0.0 \\
\hline Refrigerator & 3 & 100 & $3.0 \times 10^{2} \pm 0.57 \times 10^{2}$ & 0 & 0.0 & 0.0 & 0 & 0.0 & 0.0 \\
\hline
\end{tabular}


Table 7: Statistical analytical results of microbiological examination for examined ras cheese during processing steps of cheese production line.

\begin{tabular}{|c|c|c|c|c|c|c|c|c|c|}
\hline \multirow{2}{*}{$\begin{array}{c}\text { Control } \\
\text { points }\end{array}$} & \multicolumn{3}{|c|}{ Total coliforms } & \multicolumn{3}{|c|}{ T. Yeast } & \multicolumn{3}{|c|}{ T. mould } \\
\hline & NO & $\%$ & Mean \pm SEM & NO & $\%$ & Mean+SEM & NO & $\%$ & Mean \pm SEM \\
\hline Ras cheese & 0 & 0.0 & 0.0 & 6 & 60 & $\begin{array}{l}4.4 \times 10^{3} \pm \\
1.05 \times 10^{3}\end{array}$ & 7 & 70 & $\begin{array}{l}4.5 \times 10^{3} \pm \\
0.99 \times 10^{3}\end{array}$ \\
\hline Raw milk & 10 & 100 & $\begin{array}{r}5.25 \times 10^{4} \pm \\
0.81 \times 10^{4} \\
\end{array}$ & 7 & 70 & $\begin{array}{c}1.22 \times 10^{4} \pm \\
0.35 \times 10^{4}\end{array}$ & 6 & 60 & $\begin{array}{l}5.1 \times 10^{3} \pm \\
1.19 \times 10^{3} \\
\end{array}$ \\
\hline Water supply & 0 & 0.0 & 0.0 & 0 & 0.0 & 0.0 & 3 & 30 & $1.0 \times 10^{3} \pm 0.0$ \\
\hline Rennet enzyme & 0 & 0.0 & 0.0 & 0 & 0.0 & 0.0 & 4 & 40 & $\begin{array}{l}3.2 \times 10^{3} \pm \\
1.03 \times 10^{3}\end{array}$ \\
\hline Salt & 0 & 0.0 & 0.0 & 0 & 0.0 & 0.0 & 0 & 0.0 & 0.0 \\
\hline Air & 0 & 0.0 & 0.0 & 0 & 0.0 & 0.0 & 2 & 20 & $\begin{array}{c}5.05 \times 10^{2} \pm \\
4.9 \times 10^{2} \\
\end{array}$ \\
\hline Curd & 0 & 0.0 & 0.0 & 0 & 0.0 & 0.0 & 2 & 20 & $1.0 \times 10^{3} \pm 0.0$ \\
\hline Whey & 0 & 0.0 & 0.0 & 0 & 0.0 & 0.0 & 1 & 10 & $1.0 \times 10^{3}$ \\
\hline \multicolumn{10}{|c|}{ Swabs (Cfu/cm2) } \\
\hline Milk tank & 1 & 33.3 & $2.0 \times 10^{3} \pm 0.0$ & 1 & 33.3 & $4.0 \times 10 \pm 0.0$ & 1 & 33.3 & $5.0 \times 10 \pm 0.00$ \\
\hline Equipment & 3 & 100 & $\begin{array}{c}6.4 \times 10^{5} \pm \\
1.7 \times 10^{5}\end{array}$ & 2 & 66.6 & $\begin{array}{l}1.3 \times 10^{4} \pm \\
0.65 \times 10^{4}\end{array}$ & 3 & 100 & $\begin{array}{l}1.6 \times 10^{3} \pm \\
0.87 \times 10^{3}\end{array}$ \\
\hline Food handlers & 1 & 33.3 & $1.0 \times 10^{3} \pm 0.0$ & 0 & 0.0 & 0.0 & 3 & 100 & $\begin{array}{l}2.0 \times 10^{3} \pm \\
0.57 \times 10^{3}\end{array}$ \\
\hline Tables & 0 & 0.0 & 0.0 & 0 & 0.0 & 0.0 & 0 & 0.0 & 0.0 \\
\hline Walls & 0 & 0.0 & 0.0 & 0 & 0.0 & 0.00 & 2 & 66.6 & $\begin{array}{c}2.5 \times 10^{3} \pm \\
0.5 \times 10^{3}\end{array}$ \\
\hline $\begin{array}{r}\text { Packaging } \\
\text { Material }\end{array}$ & 0 & 0.0 & 0.0 & 0 & 0.0 & 0.00 & 0 & 0.0 & 0.0 \\
\hline Refrigerator & 0 & 0.0 & 0.0 & 0 & 0.0 & 0 & 0.0 & 0.0 & 0.0 \\
\hline
\end{tabular}

Table 8: Incidence of yeast and fungal isolates in examined ras cheese during processing steps of cheese production line collected from different sources $(\mathrm{N}=101)$.

\begin{tabular}{|c|c|c|c|c|c|c|c|c|c|c|c|c|}
\hline \multirow{3}{*}{ Control points } & \multicolumn{6}{|c|}{ Yeast species } & \multicolumn{6}{|c|}{ Mould species } \\
\hline & \multicolumn{2}{|c|}{ C. albicans } & \multicolumn{2}{|c|}{ C. tropical } & \multicolumn{2}{|c|}{ Rhodotorula } & \multicolumn{2}{|c|}{ A. flavus } & \multicolumn{2}{|c|}{ A. niger } & \multicolumn{2}{|c|}{ Penicillium } \\
\hline & $\overline{\text { No }}$ & $\%$ & NO & $\%$ & No & $\%$ & No & $\%$ & No & $\%$ & No & $\%$ \\
\hline Ras cheese & 1 & 10 & 2 & 20 & 5 & 50 & 2 & 20 & 5 & 50 & 3 & 30 \\
\hline Raw milk & 2 & 20 & 5 & 50 & 5 & 50 & 3 & 30 & 4 & 40 & 2 & 20 \\
\hline Water supply & 0 & 0 & 0 & 0 & 0 & 0 & 1 & 10 & 2 & 20 & 2 & 20 \\
\hline Rennet enzyme & 0 & 0 & 0 & 0 & 0 & 0 & 1 & 10 & 2 & 20 & 3 & 30 \\
\hline Salt & 0 & 0 & 0 & 0 & 0 & 0 & 0 & 0 & 0 & 0 & 0 & 0.0 \\
\hline Air & 0 & 0 & 0 & 0 & 0 & 0 & 0 & 0 & 2 & 20 & 2 & 20 \\
\hline Curd & 0 & 0 & 0 & 0 & 0 & 0 & 0 & 0 & 2 & 20 & 1 & 10 \\
\hline Whey & 0 & 0 & 0 & 0 & 0 & 0 & 1 & 10 & 1 & 10 & 0 & 0 \\
\hline \multicolumn{13}{|c|}{ Swaps } \\
\hline Milk tank & 0 & 0 & 1 & 33.3 & 1 & 33.3 & 0 & 0 & 1 & 33.3 & 1 & 33.3 \\
\hline Equipment & 1 & 33.3 & 1 & 33.3 & 2 & 66.6 & 1 & 33.3 & 2 & 66.6 & 2 & 66.6 \\
\hline Food handlers & 0 & 0 & 0 & 0 & 0 & 0 & 1 & 33.3 & 2 & 66.6 & 2 & 33.3 \\
\hline Tables & 0 & 0 & 0 & 0 & 0 & 0 & 0 & 0 & 0 & 0 & 0 & 0 \\
\hline Walls & 0 & 0 & 0 & 0 & 0 & 0 & 0 & 0 & 2 & 66.6 & 2 & 66.6 \\
\hline Packaging Material & 0 & 0 & 0 & 0 & 0 & 0 & 0 & 0 & 0 & 0 & 0 & 0 \\
\hline Refrigerator & 0 & 0 & 0 & 0 & 0 & 0 & 0 & 0 & 0 & 0 & 0 & 0.0 \\
\hline
\end{tabular}




\section{DISCUSSION}

During the processing line in dairy facilities, hygienic practices and control \& assurance activities are employed to control microbial development and crosscontamination that could come from contaminated surfaces and/or food handlers' hands. (Opiyo et al., 2013).

Results recorded in Table (1) revealed that the mean value of Fat content, moisture content, salt, $\mathrm{pH}$ and acidity \% of examined damietta cheese were $18.96 \pm$ $0.25,43.27 \pm 0.37,5.01 \pm 0.25,4.43 \pm$ 0.099 and $1.36 \pm 0.25$, respectively. Nearly similar results of acidity percent and salt content was reported by (Abo El-Makarem

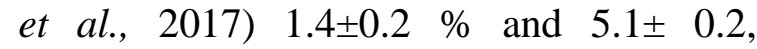
respectively. While lower acidity percent was obtained by (Salama et al., 2015) 0.72 \pm $0.039 \%$. Relative variations in acidity percent of the examined damietta cheese samples may be attributed to variation in manufacturing process, ripening periods, temperature of storage and/ or age of cheese samples (Mohamed, 2016).

Because the microflora can hydrolyze lactose into lactic acid, Damietta cheese had the greatest $\mathrm{pH}$ values $(6.05$ - 6.32) for fresh cheese and declined significantly as the ripening progressed, $\mathrm{pH}$ values gradually decreased during 6 months of ripening to reach $(3.05-3.52)$. In addition, the author reported moisture content and fat content of damietta cheese during first month of storage was $46.43 \pm 0.12$ and 22.99 \pm 0.08 (Hamad, 2015).

The purpose of a hazard analysis for cheese manufacturing is to identify numerous dangers in various raw materials and processing steps, as well as to propose control solutions for the hazards (Mauropoulos and Arvanitoyannis, 1999). The main source of biological risks in cheese factory is raw milk which is the most important source of pathogenic microorganisms (Nasr, 2018).
The above mentioned results in Tables $(2,3)$ indicating that $70,70,50$ and $20 \%$ of fresh damietta cheese were contaminated with total bacterial, Staph. aureus, total yeast and total mould with mean values of $5.57 \times 10^{4} \pm 1.07 \times 10^{4}, 1.06 \times 10^{4} \pm 0.82 \times 10^{4}$, $4.14 \times 10^{4} \pm 1.27 \times 10^{4}$ and $1.3 \times 10^{3} \pm 0.5 \times 10^{3}$ $\mathrm{Cfu} / \mathrm{g}$, respectively. While the fresh damietta cheese was free from coliforms and enterobacteriaceae count due to efficient pasteurization and good manufacturing practices (Singh and Prakash, 2008). The finished cheese product was found to be inappropriate according to Egyptian Standards (ES: 10083/2005). In the same direction, lower incidence in fresh damietta cheese was obtained by (Osama et al., 2014) who indicated that $4.5,22.5$ and $4.5 \%$ of examined samples were contaminated with Staph. aureus, yeast and mould count. On the contrary, (Osama et al., 2014) mentioned that $18 \%$ of damietta cheese was contaminated with coliforms ranged from 23 to $24 \times 10 \mathrm{Cfu} / \mathrm{gm}$.

In this investigation, the higher staph. aureus count could be attributed to a poor worker personal hygiene, which resulted in contamination of cheese from the skin, mouth, or handling of the meal, but improperly cleaned utensils or equipment could also be a source of contamination (ICMSF, 1980).

Higher yeast counts in the current study have resulted from insufficient processing. Yeast spoilage costs the cheese industry a lot of money since it causes unwanted changes, like sliminess, red colour, and yeasty flavour (Sarais et al., 2009). Fruity, bitter, or yeasty off tastes, gas production, colouring changes, and texture are the most common spoilage yeast faults. Continued lactose fermentation may result in increasing acidity, gassiness, and fruity flavors, whereas continued protein and fat hydrolysis may result in bitter and rancid flavors, as well as a softening of product texture (Soloiman and Aly, 2011). Molds and yeasts in cheese are undesirable 
because they can develop at a wide variety of temperatures and $\mathrm{pH}$ levels, causing the product to deteriorate. Their number is employed as a measure of the product's storability and sanitary quality (Osama $e t$ al., 2014).

Recorded results in Tables $(2,3)$ showed higher load of total bacterial count $5.38 \times 10^{7} \pm 1.09 \times 10^{7} \mathrm{Cfu} / \mathrm{ml}$ followed by curd $\left(1.5 \times 10^{4} \pm 0.71 \times 10^{4} \mathrm{Cfu} / \mathrm{ml}\right)$ and salt $\left(2.2 \times 10^{3} \pm 0.47 \times 10^{3} \mathrm{Cfu} / \mathrm{ml}\right)$, contained $3.8 \times 10^{4} \pm 1.01 \times 104, \quad 5.8 \times 10^{4} \pm 4.06 \times 10^{4}$, $1.4 \times 10^{4} \pm 0.41 \times 10^{4}, \quad 2.04 \times 10^{4} \pm 0.56 \times 10^{4}$ and $6.8 \times 10^{3} \pm 1.68 \times 10^{3} \mathrm{Cfu} / \mathrm{ml}$ Staph. aureus, total enterobacteriaceae, total coliforms, total yeast and total mould. Obtained results indicate that raw milk produced and handled under neglected hygienic measure, this result was in agreement with (Nasr, 2018) who applied hazard analysis on raw milk used in small factory of fresh damietta cheese and found that higher bacterial count $\left(2.5 \times 10^{8}\right)$ in raw milk was observed, total coliforms $\left(4.5 \times 10^{3}\right)$ and total fungi $\left(8.5 \times 10^{5}\right) \mathrm{Cfu} / \mathrm{ml}$ thereby suggested that raw milk produced under lack hygienic practices.

On the other hand, while rennet enzyme and salt had $4.4 \times 10^{2} \pm 1.3 \times 10^{2}, \quad 2.2 \times 10^{3} \pm$ $0.47 \times 10^{3} \mathrm{cfu} / \mathrm{ml}$ of total bacterial count, respectively, they were not as dangerous as raw milk since additional bacteria were not discovered in rennet enzyme, which was also added in a modest amount for coagulation. The water supply and air, on the other hand, contained low levels of total bacteria, yeast, and mould. (Nasr, 2018) revealed that rennet enzyme contains $8.3 \times 10^{6}, 1.2 \times 10^{6}$ and $2.5 \times 10^{2}$ total bacterial count, mould count and total coliforms $/ \mathrm{ml}$, respectively.

Swabs from containers, utensils, food handlers, tables, walls, packing material, and refrigerators were microbiologically tested since they are highly major sources of microbial contamination of cheese product throughout processing processes. The results in (Tables 2, 3) revealed that, total bacterial count was not detected in all swabs between $7.6 \times 10 \pm 2.4 \times 10$ to $4.7 \times 10^{7} \pm$ $2.4 \times 10^{7} \mathrm{Cfu} / \mathrm{Cm}^{2}$; total staph aureus was detected in $100 \%$ of examined swabs from equipment, food handler and walls. Enterobacteriaceae and coliforms count were detected in $100 \%$ in examined swabs from equipment with mean values $2.0 \times 10^{4} \pm 0.8 \times 10^{5}$ and $2.7 \times 10^{5} \pm 1.8 \times 10^{5}$ $\mathrm{Cfu} / \mathrm{ml}$, respectively while swabs from food handler and walls, enterobacteriaceae and coliforms equally were detected in $33.33 \%$ of examined swabs. Total yeast and mould were detected in swabs sample collected from milk tank, equipment, food handlers and walls at different incidence.

The obtained result indicating that coliforms was not found in tables, packaging material and refrigerators swabs and found in other swabs samples with higher count in swabs from equipment with mean values $2.7 \times 10^{5} \pm 1.8 \times 10^{5} \mathrm{Cfu} / \mathrm{Cm}^{2}$. In the same direction, (Nasr, 2018) reported that coliforms were not found in wall and refrigerator swabs during application of HACCP in small damietta factory.

Results recorded in Table (4) revealed that the most prevalent yeast species isolated during processing steps of Damietta cheese production line collected from different sources was Rhodotorula 66.6, 50 and $40 \%$ from milk tank swab, raw milk and damietta cheese, respectively, followed by candida tropicalis from swab samples of milk tank, equipment and wall. While the most prevalent fungal isolates were $\mathrm{A}$. Niger and penicillium species from swab samples from equipment $(66.6 \%)$ and wall (66.6\%), respectively, followed by A. Niger from raw milk (40\%).

In the same way, (Hameed, 2016) found that Candida albicans was clearly the dominant yeast in examined Damietta cheese Candida, while Cladosporium cladosporidis was the most prevalent mould species isolated from the same cheese sample. In addition, (Vinayananda et al., 2018) reported higher fungal count in 
examined water, hand swabs, cheese molds and utensils, tank swabs and air samples in dairy plant.

Toxic metabolites are secreted by Candida species, which induce a variety of symptoms (Silva, 2010). Candida species' pathogenicity in the gastrointestinal tract is linked to adhesion factors that mediate yeast attachment to cell surfaces, as well as the production of lipase, protease, and phospholipase, all of which aid in their propagation and invasion (Schulze and Sonnenborn, 2009). Bitterness and rancidity of cheese may be caused by the growth of such genera. Penicillium species have been linked to the softening of cheese surfaces. According to (Minervini et al., 2001), they have been linked to lung infections, urinary tract infections, and death in humans. Many species of Aspergillus, Cladosporium, Penicillium, and Fusarium induce keratoconjunctivitis in humans, while Aspergillus Niger causes otomycosis and allergy conditions, according to (Nielsen et al., 1998).

Mentioned results in Table (5) revealed that the mean value of Fat content, moisture content, salt, $\mathrm{pH}$ and acidity $\%$ of examined ras cheese was $47.79 \pm 0.17,32.23 \pm 0.45$, $2.35 \pm 0.036,4.96 \pm 0.066$ and $1.76 \pm 0.06$, respectively. (Osman et al., 2011) reported the mean values of fat, moisture, acidity and salt content of examined ras cheese collected from Assiut markets were 52.21, 35.96, 1.51 and 3.59, respectively. In addition, (El-Fadaly et al., 2015) reported moisture content and fat content of fresh ras cheese were $38.86 \pm 1.21, \quad 39.33 \pm 0.22$, respectively. In addition, (El-Tahra et al., 2017) indicated that fat and $\mathrm{pH}$ of ras cheese were 28.12 and 5.71, respectively. The wide variations in the chemical composition of the present study and those from previous studies reflect the need of renewal of the standards procedures in selecting the raw material used, the conditions of manufacture and storage of this cheese.
Tabulated data in Tables ( 6 and 7) revealed that 100, 100, 60 and $70 \%$ of examined fresh ras cheese was contaminated with total bacteria, Staph. aureus, total yeast and total mould with mean values of $2.7 \times 10^{5} \pm$ $0.76 \times 10^{5}, 4.6 \times 10^{4} \pm 0.86 \times 10^{4}, 4.4 \times 10^{3} \pm$ $1.05 \times 10^{3}$ and $4.5 \times 10^{3} \pm 0.99 \times 10^{3} \mathrm{Cfu} / \mathrm{g}$, respectively. While examined ras cheese was free from enterobacteriaceae and coliforms. In the same direction, (El-Leboudy et al., 2014) could be detected higher level of bacterial count in fresh ras cheese $1.15 \times 10^{8} \pm 3.04 \times 10^{7} \mathrm{Cfu} / \mathrm{g}$, in addition, Staph. aureus and total yeast \& mould were $1.64 \times 103 \pm 3.66 \times 102$ and $4.60 \times 10 \pm 0.979 \times 10 \mathrm{Cfu} / \mathrm{g}$, respectively. On the contrary, the same authors could detect coliforms in fresh damietta cheese with mean value of $1.88 \times 10^{3} \pm 0.81 \times 10^{2}$ $\mathrm{Cfu} / \mathrm{g}$.

Results in Tables (6 and 7) showed that microbiological analysis of raw materials used during manufacturing of ras cheese, where the 100,90,30, 100, 70 and $60 \%$ of raw milk contaminated with total bacterial count, staph aureus, enterobacteriaceae, coliforms count, yeast and mould count with mean values of $1.3 \times 10^{7} \pm 0.39 \times 10^{7}$, $1.8 \times 10^{4} \pm 0.71 \times 10^{4}, \quad 3.4 \times 10^{5} \pm 0.30 \times 10^{5}$, $5.25 \times 10^{4} \pm 0.81 \times 10^{4}, 1.22 \times 10^{4} \pm 0.35 \times 10^{4}$, and $5.1 \times 10^{3} \pm 1.19 \times 10^{3} \mathrm{Cfu} / \mathrm{g}$, respectively. A higher quantity of these indicator organisms indicates that raw milk was made and handled without following proper hygienic procedures, or that there were no cooling facilities available during transportation. These findings were supported by (El-Leboudy et al., 2014) who detected higher bacterial, and coliforms count in raw milk used for manufacture of ras cheese with mean values of $1.11 \times 10^{7} \pm$ $0.24 \times 10^{7}$ and $8.24 \times 10^{4} \pm 1.68 \times 10^{4} \mathrm{Cfu} / \mathrm{g}$, respectively. In addition, the same author reported low level of Staph. aureus and yeast \& mould count in received raw milk used for ras cheese manufacturing with mean value of $7.77 \times 10^{2} \pm 4.33 \times 10^{2}$ and $4.10 \times 10^{2} \pm 2.0 \times 10^{2} \mathrm{Cfu} / \mathrm{g}$, respectively. 
On the other hand, other raw materials were contaminated with total bacterial count where higher count in salt $\left(1.0 \times 10^{3} \pm\right.$ $\left.0.57 \times 10^{3} \mathrm{Cfu} / \mathrm{ml}\right)$ followed by rennet enzyme $\left(8.4 \times 10^{2} \pm 5.4 \times 102 \mathrm{Cfu} / \mathrm{ml}\right)$ then curd $5.0 \times 10^{2} \pm 1.1 \times 10^{2} \mathrm{Cfu} / \mathrm{ml}$. Another indicating organism including (Staph. aureus, coliforms, enterobacteriaceae and yeast) were not detected in raw material. Our results indicating the most important source of contamination is raw milk used from manufacturing especially that raw milk produced under bad hygienic conditions. Higher bacterial count was obtained by (El-Leboudy et al., 2014) who reported that mean value of bacterial count in rennet enzyme and cheese curd was 1.70 $\times 10^{5} \pm 1.26 \times 10^{5}$ and $1.24 \times 10^{6} \pm 1.19 \times 10^{6}$ $\mathrm{Cfu} / \mathrm{g}$, respectively. On the contrary, the same authors detected coliforms and staphylococcus count in rennet enzyme and ras cheese curd at mean values $\left(5.33 \times 10 \pm 1.85 \times 10, \quad 4.15 \times 10^{2} \quad \pm 1.82 \times 10^{2}\right)$ and $\left(1.37 \times 10^{5} \pm 1.30 \times 10^{5}, \quad 1.72 \times 10^{2} \pm\right.$ $\left.0.42 \times 10^{2}\right) \mathrm{Cfu} / \mathrm{g}$, respectively.

Above-mentioned results in (Tables 6 and 7 ) indicated that, total bacterial count was detected in all swabs ranged between $2.0 \times 10 \pm 0.5 \times 10$ to $4.07 \times 10^{7} \pm 2.3 \times 10^{7}$ $\mathrm{Cfu} / \mathrm{Cm}^{2}$, higher total bacterial count was observed in equipment swabs $4.07 \times 10^{7} \pm$ $2.3 \times 10^{7} \mathrm{Cfu} / \mathrm{cm}^{2}$ followed by table swabs $2.3 \times 10^{4} \pm 0.88 \times 10^{4} \mathrm{Cfu} / \mathrm{cm}^{2}$ and food handler swabs $1.2 \times 10^{4} \pm 0.14 \times 10^{4} \mathrm{Cfu} / \mathrm{cm}^{2}$. in the same direction, higher staphylococcal count was observed in equipment swabs $5.3 \times 10^{5} \pm 1.1 \times 10^{5}$ followed by wall swabs $1.3 \times 10^{4} \pm 0.96 \times 10^{4}$ and food handler swab $1.4 \times 10^{3} \pm \quad 0.29 \times 10^{3} \quad \mathrm{Cfu} / \mathrm{cm}^{2}$. Enterobacteriaceae was detected only in food handler swab with mean value $5.9 \times 10^{5} \pm 2.4 \times 10^{5} \mathrm{Cfu} / \mathrm{cm}^{2}$. Coliforms were detected only in milk tank, equipment and food handler swabs with higher count in equipment swabs $6.4 \times 10^{5} \pm 1.7 \times 10^{5}$ $\mathrm{Cfu} / \mathrm{cm}^{2}$. Finally, total yeast and mould were detected in milk tank, equipment, food handler and wall swabs with higher level in equipment swabs. Total coliforms, enterobacteriaceae, yeast and mould
Packaging material and refrigerator swabs could not be detected in packaging material and refrigerator swabs. Higher bacterial count in food handler swab was obtained by (El-Leboudy et al., 2014) who reported that mean value of total bacterial count from worker's hand in ras cheese factory was $9.90 \times 10^{8} \pm 6.21 \times 10^{8} \mathrm{Cfu} / \mathrm{cm}^{2}$, in addition, they reported that total coliforms, total staphylococcal and total yeast \& mould were $9.14 \times 10^{3} \pm 2.48 \times 10^{3}, 1.13 \times 10^{3} \pm$ $5.53 \times 10^{3}$ and $1.46 \times 10^{2} \pm 0.38 \times 10^{2}$ $\mathrm{Cfu} / \mathrm{cm}^{2}$, respectively.

Results recorded in table (8) revealed that the most prevalent yeast species isolated during processing steps of ras cheese production line collected from different sources was Rhodotorula $66.6 \%$ from equipment swab followed by candida tropicalis and Rhodotorula from raw milk samples with incidence rate $50 \%$ for both. while the most prevalent fungal isolated were A. Niger and penicillium species from equipment, food handler and wall swabs $(66.6 \%)$ followed by A. Niger from raw milk $(50 \%)$. The obtained results were similar to (Abd El Tawab et al., 2020) reported that Rhodotorula was the most prevalent yeast isolated from raw milk at indicine rate $42.8 \%$ followed by candida tropicalis $14.3 \%$. also reported that Aspergillus flavus was the most prevalent mould species isolated from raw milk at incidence rate $42.8 \%$ followed by Asp. niger $28.6 \%$. In addition, (El-Fadaly et al., 2015) reported that Aspergillus oryzae, Aspergillus glaucus, Aspergillus ochraceus and Aspergillus niger were the prevalent fungal isolated from Ras cheese samples collected from six different factories was observed in all factories.

Fungal contamination of raw milk and cheese occur as a result of wide distribution of fungal spores in un sanitized environment which lead to milk and cheese contamination during their production, transportation, manufacturing and storage. Also, fungal contamination may be resulted from contaminated air, bad environment in 
some factories as, uncontrolled sterilization, use of low quality milk, unhygienic utensils, equipment, workers, clothes or recontamination after pasteurization, transportation or storage (Younis et al., 2016).

It is important to maintain prerequisite programs, such as good cleaning and disinfection of surfaces and equipment, in order to effectively implement the HACCP system and produce safe food. Estimating the degree of contamination during production and evaluating the success of cleaning and disinfection processes require determining the total microbial number on the surfaces of utensils, work surfaces, and other equipment in contact with food (ISO/FDIS, 2004).

\section{CONCLUSION AND RECOMMENDATION}

Finally, because of their nutrient content and lengthy storage time, cheese might be regarded an excellent substrate for bacterial development. Several processes in the manufacturing process can result in bacterial contamination. The findings reveal unsanitary circumstances that existed during production, distribution, sale, or storage. The HACCP system is an effective way for assuring the safety and satisfaction of food products for consumers. This boosts the company's competitiveness and strengthens its positions. It is suggested that the application of Good Manufacturing Practices (GMP), Good Hygienic Practices (GHP), and the Hazard Analysis and Critical Control Point (HACCP) system, which seeks to give HACCP guidelines, be used to improve product quality and safety.

\section{REFERENCES}

Abd El Tawab, A.A.; El-Hofy, F.I.; ELdiasty, E.M.; Abo-Hamdah, E. and AlKhayat, M. (2020): Diversity of some food borne fungi associated with raw milk and some cheese in Egypt. Benha Veterinary Medical Journal 38 (1): 48-51

Abo El-Makarem, H.S.; Saber, A.S. and ElAsuoty, M.S. (2017): Chemical and microbiological evaluation of some different soft cheese. Assiut Veterinary Medical Journal Assiut Vet. Med. J. 63 (154): 10-19

Abou-Donia, S.A. (2002): Recent development in Ras cheese research: A review. Egyptian J. Dairy Sci. 30: 155-160.

AOAC, "Association of official Agriculture Chemists" (2000): Association of official Analytic Chemists. Official Methods of Analysis Association of Official Agriculture Chemists. 17th ed. Wisconsin: Georgia Banta Co. Inc.

AOAC, "Association of official Agriculture Chemists" (2012): Official Methods of Official Analysis Chemists. 19th ed. William Hornitz, (Ed) pub Association of official Agric. Chemists, Washington, DC, USA. https://doi.org/10.5962/bhl.title.44636

APHA "American Public Health Association” (2004): Standards Methods for the Examination of Dairy Products. 17th ed., H. Michael Wehr and Joseph F. Frank, editors. American Public Health Association, Washington, DC 2001, USA.

APHA, "American Public Health Association” (1992): Standard Methods for the Examination of Dairy Products. 14th Ed., Washington, D.C.

Awad, A.H. and Mawla, H.A. (2012): Sedimentation with the Omeliansky formula as an accepted technique for quantifying airborne fungi. Pol $\mathrm{J}$ Environ Stud, 21: 1539- 1541.

Ayad, E.H.E.; Awad, S.; El-Attar, A.; De Jong, C. and El-Soda, M. (2004): Characterization of Egyptian Ras cheese. 2. Flavour formation. Food Chemistry, 86: 553-561.

EFSA "European Food Safety Authority" (2007): The community summary 
report on trends and sources of zoonoses, zoonotic agents, antimicrobial resistance and food borne outbreaks in the European Union in 2006. Available at: www.efsa.europa.eu/EFSA/efsalocale1178620753812_11787131291 2.htm. Accessed 12 June 2012.

Egyptian Standards (2005): Soft cheese: Domiati cheese. ES: 1008- 3 / 2005

El-Fadaly, H.A.; Hamad, M.N.F.; El-Kadi, S.M.L. and Habib, A.A. (2015): Effect of Clove Oil on Physicochemical and Microbiological Characteristics of Egyptian Ras Cheese (Romy) during Storage. International Journal of Food Science and Nutrition Engineering, 5(1): 15-23.

El-Leboudy, A.A.; Amer, A.A. and Micheal, R.Y. (2014): Assessment of sanitary measures of ras cheese in manufacturing dairy plant in Alexandria Governorate. Alexandria Journal of Veterinary Sciences, 40:87-94.

El-Tahra, M.A.A.; Reyad, M.Y.; AbdelKader, Y.I. and Farag, A.M.K. (2017): Implementation of the Hazard Analysis Critical Control Point (HACCP) System for Processed Cheese Production Line. J. Food and Dairy Sci., Mansoura Univ., Vol. 8(2): 121 - 125, 2017.

Evans, J.A.; Russell, S.L.; James, C. and Corry, J.E.L. (2004): Microbial contamination of food refrigeration equipment. J. Food Eng. 62: 225-232.

Frey, D.; Old, R.J. and Bridger, R.C. (1979): A colour atlas of pathogenic fungi. Walfe Medical Publication Ltd, Holland.

Fuster-Valls, N.; Hernandez-Herrero, M.; Marin-de-Mateo, M. and RodriguezJerez, J.J. (2008): Effect of different environmental conditions on the bacteria survival on stainless steel surfaces. Food Control 19: 308-314.

Girgis, E.S.; Hassan, N.A.; Youssef, L.M. and Harby, S.I. (1992): Some chemical and microbiological properties of Ras cheese in the
Egyptian market. Egyptian J. Dairy Sci.20: 273-287.

Grujić, R.; Ivanović, M. and Antonić, B. (2010): Implementation of Food Safety Management System in Food Production and Handling in Northern Serbia. Quality of Life, 1 (2-4), 114120.

Hamad, M.N.F (2015): Comparative study between traditional Domiati cheese and recombined Feta cheese. Indian J. Dairy Sci. 68(5): 442-452.

Hameed, K.G.A. (2016): Fungal diversity in different types of cheese and the effect of natamycin on their survival during Feta cheese manufacture and storage. Journal of Advanced Veterinary and Animal Research, 3(3): 214-220.

Henning, D.R.; Flowers, R.; Reiser, R. and Ryser, E.T. (2004): Pathogens in milk and milk products: Standard methods for the examination of dairy products (Chapter 5). APHA, Washington DC, USA.

ICMSF "International Committee Microbiological Specification for foods" (1980): Effect of processing on microorganisms. Further contamination p.419. Microbial Ecology of food. Food commodities Academic press Univ. of Toronto press, Toronto, Canada

ISO "International Organization for Standardization" (2004): Microbiology of food and animal feeding stuffs- Horizontal methods for the detection and enumeration of Enterobacteriaceae - Part 2: Colonycount method. 21528-2.

ISO, "International Organization for Standardization (2004): Microbiology of food and animal feeding stuffs. Horizontal methods for sampling techniques from surfaces using contact plates and swabs. ISO 18593:2004. International Organization for Standardization, Geneva.

ISO, "International Organization for Standardization" (2004): Milk and 
milk products-Enumeration of colony forming units of yeasts and/or moulds-Colony-count technique at $25^{\circ}$ C. ISO 6611:2004, International Standard Organization, Geneva, Switzerland. https://www.iso.org/ standard/40473.html.

ISO, "International Organization for Standardization" (2010):

Microbiology of food and animal feeding stuffs - Preparation of test samples, initial suspension and decimal dilutions for microbiological examination - Part 5: Specific rules for the preparation of milk and milk products $6887-5$.

ISO/FDIS (2004): Microbiology of food and animal feeding stuff-Horizontal methods for sampling techniques from surfaces using contact plates and swabs, 18593, 2004.

Jones, S.L.; Parry, S.M.; O'Brien, S.J. and Palmer, S.R. (2008): Are staff management practices and inspection risk ratings associated with foodborne disease outbreaks in the catering industry in England and Wales? J. Food Prot. 71: 550-557.

Koburger, J.A. (1970): Fungi in Foods.1Effect of inhibitor and incubation temperature on enumeration. J. Milk and Food Technol., 33(10): 433-434.

Kriger, V.R. (1984): The yeasts: A taxonomic study. 3rd Ed.

Amsterdam, Elsevier.

Ledenbach, L.H. and Marshall, R.T. (2009): Microbiological Spoilage of Dairy Products. Compendium of the microbiological spoilage of food and beverages. Pp.41-67.

Mauropoulos, A.A. and Arvanitoyannis, I. (1999): Implementation of hazard analysis critical control point to Feta and Manouri cheese production lines. Food Control, 10: 213-219.

McMeekin, T.A. (2003): Detecting Pathogens in Food. Woodhead Publishing Limited Abington Hall, Abington Cambridge, England, pp: 319.
Minervini, F.; Montagna, M.T.; Spilotros, G.; Monaci, L.; Santacroce, M.P. and Visconti, A. (2001): Survey on mycoflora of cow and buffalo dairy products from Southern Italy. International Journal of Food Microbiology, 69: 141-146.

Mohamed, Z.A. (2016): Incidence of mycobiota in some dairy products and its public health hazards. Master thesis. Fac. Vet. Med., Alex. Univ. Egypt.

Nasr, N.F. (2018): Minimizing of Microbiological Hazards in Small Cheese Plants by Application of Food Safety Programs. $1^{\text {st }}$ International Conference of the Egyptian Society of Food Safety, Sharm El-Sheikh, Egypt, February 15-18, 2018

Nielsen, M.S.; Frisvad, J.C. and Nielsen, P.V. (1998): Protection by fungal starter against growth and secondary metabolite production of fungal spoilers of cheese. International Food Microbiology, 42: 91-99.

Opiyo, B.A.; Wangoh, J. and Njage, P.M.K. (2013): Microbiological Performance of Dairy Processing Plants Is Influenced by Scale of Production and the Implemented Food Safety Management System: A Case Study. Journal of Food Protection, 76 (6): 975-983.

Osama, M.S.; Gamal, A.I.; Nabil, F.T.; Baher, A.M.E.; Kawther, E.; Hala, M.F. and Moussa, M.A.S. (2014): Prevalence of some pathogenic microorganisms in factories Domiati, Feta cheeses and UHT milk in relation to public health sold under market conditions in Cairo. Int. J. Chem. Tech. Res., 6(5): 2807-2814.

Osman, D.M.; Shahin, Y.H.; Abd El-Galil, H.A. and Mohamed, A.M. (2011): Chemical and Microbiological Characteristics of Ras Cheese Collected from Assiut Markets. Assiut J. of Agric. Sci., 42(3): 47-54.

Pitt, J.I. and Hocking, A.D. (2009): Fungi and Food spoilage. 3rd Ed. Published 
by Springer Dordrecht Heidelberg, London, New York.

Raper, K.B. and Fennel, D.T. (1965): The genus Aspergillus. The Williams and Wilkins co., Baltimore

Salama, E.M.; Saad, A.H.; Enan, G.A. and Suzan, I.Y. (2015): Incidence and Biocontrol of Staphylococcus aureus in some milk products. 2nd Conference of Food Safety, Suez Canal University, Faculty of Veterinary Medicine Volume I August 2015 Page 29-35.

Samson, R.A. (1979): Aspergilli described since 1965. Studies in Mycology No.18.

Sarais, I.; Piussi, D.; Aquili, V. and Stecchini, M.L. (2009): The behavior of yeast populations in stracchino cheese packaged under various conditions. J. Food Protect. 59(4): 541-544.

Schulze, J. and Sonnenborn, U. (2009): Yeasts in the gut: from commensals to infectious agents. Deutsches Ärzteblatt International, 106: 837842.

Silva, R.F. (2010): Infecções fúngicas em imunocomprometidos. Jornal Brasileiro de Pneumologia, 36: 142147.

Singh, P. and Prakash, A. (2008): Isolation of Escherichia coli, Staphylococcus aureus and Listeria monocytogenes from milk products sold under market conditions at Agro Region. Acta Agricultural Slovenica, 92: 83-88.

Soloiman, N.S.M. and Aly, S.A. (2011): Occurrence and identification of yeast species isolated from Egyptian Karish cheese. Journal of Yeast and Fungal Research, 2(4): 59-64.

Tibor, D. and Larry, R.B. (1996): Handbook of food spoilage yeasts. $1^{\text {st }}$ Edition (Contemporary Food Science) by CRC Press, Boca Raton, New York, London and Tokyo.

Tsalo, E.; Drosinos, E.H. and Zoiopoulos, $P$. (2007): Impact of poultry slaughterhouse modernization and updating of food safety management systems on the microbiological quality and safety of products. Food Control 19: 423-431.

Vinayananda, C.; Deepak, S. and Elango, A. (2018): Analysis of microbial quality of the air in meat and dairy plants by impaction technique. Bull Environm Pharmacol Life Sci, 7: 713.

Walker, E.; Pritchard, C. and Forsythe, S. (2003): Food handlers' hygiene knowledge in small food business. Food Control 14: 339-343.

WHO, "World Health Organization" (2007): Food safety and food borne illness. Factsheet 237. Available at: www.who.int/mediacentre/factsheets/ fs237/en/index.html. Accessed 13 June 2012.

Younis, G.; Ibrahim, D.; Awad, A. and ElBardisy, M.M. (2016): Determination of aflatoxin M1and ochratoxin A in milk and dairy products in supermarkets located in Mansoura City, Egypt. Advances in Animal and Veterinary Sciences 4(4): 114- 121.

Zhang, X.; Kilmer, R. and Muhammad, A. (2003): A descriptive analysis of Egypt and Saudi Arabia who import United States dairy products. Monograph MGTC 03-8. International Agricultural Trade and Policy Center, Institute of Food and Agricultural Sciences, University of Florida, Gainesville, pp: 1-51. 


\section{تأثير تطبيق نظام الهاسب على المخاطر الميكروبيولوجية المختلفة في مصانع الجبن \\ هناء فتحى سلامة ، أمل عبل المنعم ، محمد سعيد الاسيوطى}

E-mail: hsalama689@gmail.com Assiut University web-site: www.aun.edu.eg

نظر الكثرة الثكاوى من بعض منتجي الجبن بمحافظة البحيرة مصر, حول وجود مشاكل في منتجات الجبن

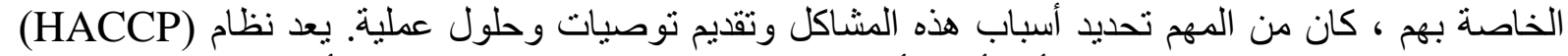

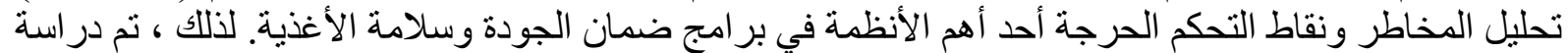

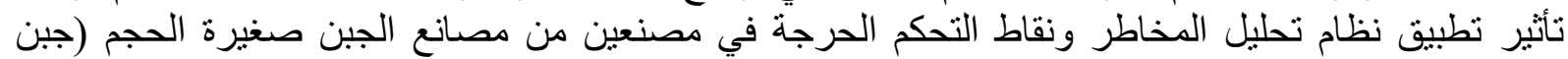

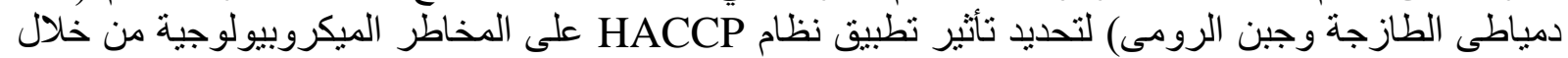
تحديد الحمل البكتيري لنقاط التحكم الحرجة (CCPs). أظهرت النتائج أن اللبن الخام والمسحات من المعدات

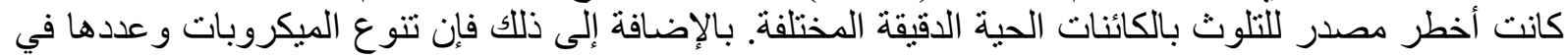

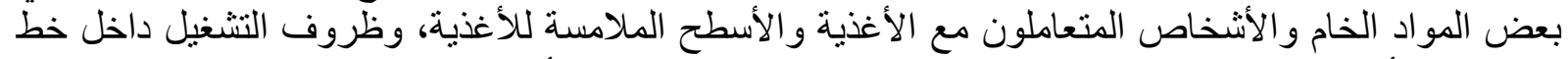

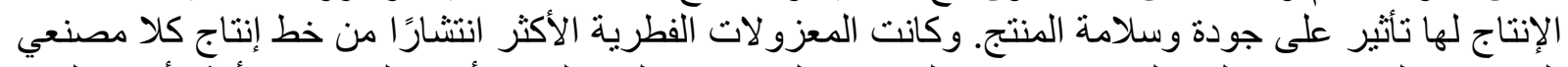

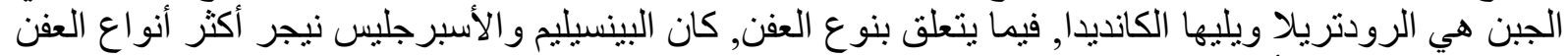

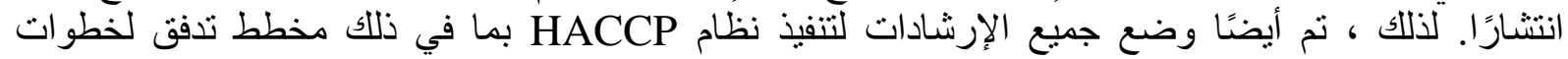

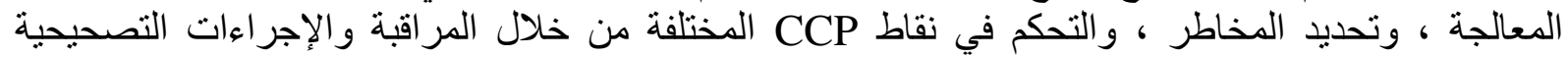
و التحقق من خطة HACCP وكذلك حفظ السجلات. 